\title{
大行程三平动柔性微定位平台的设计分析及优化
}

\author{
曹 毅 ${ }^{1,2}$ 王保兴 ${ }^{1}$ 孟 刚 $^{1}$ 林 苗 $^{1}$ 张 洪 $^{1}$
}

(1. 江南大学机械工程学院 无锡 214122;

2. 江南大学江苏省食品先进制造装备技术重点实验室 无锡 214122)

摘要: 为设计具有毫米级运动行程、良好的静、动态特性且结构多样的三维平动柔性微定位平台。首先, 提出两种新型的多 自由度大行程柔性运动副。其次, 基于该运动副, 给出三种新型大行程空间平动柔性微定位平台实例。然后, 采用非线性方 法建立平台力位移关系、输入耦合及丢失运动的理论模型, 并采用柔度矩阵法建立平台的刚度及固有频率模型。随后, 以提 高平台的静、动态性能为目标对平台进行参数寻优。最后对平台进行有限元分析，验证理论模型的正确性。根据理论与仿真 结果, 平台一阶固有频率为 $49.6 \mathrm{~Hz}$, 在 $1 \mathrm{~mm}$ 运动行程内, $x 、 z$ 轴方向的丢失运动分别小于 $0.32 \% 、 0.7 \%$, 输入耦合小于 $1.2 \%$ 且输出完全解耦。为空间平动柔性微定位平台的设计、分析及优化提供了一种系统化的研究方法。

关键词: 柔性机构; 大行程; 结构设计; 非线性分析; 参数寻优

中图分类号: V414; TH122

\section{Design Analysis and Optimization of Large Range Spatial Translational Compliant Micro-positioning Stage}

\author{
CAO Yi ${ }^{1,2}$ WANG Baoxing $^{1} \quad$ MENG Gang $^{1} \quad$ LIN Miao $^{1}$ ZHANG Hong ${ }^{1}$ \\ (1. School of Mechanical Engineering, Jiangnan University, Wuxi 214122; \\ 2. Jiangsu Key Laboratory of Advanced Food Manufacturing Equipment and Technology, \\ Jiangnan University, Wuxi 214122)
}

\begin{abstract}
In order to design spatial translational compliant micro-positioning stages with millimeter-scale motion range, excellent static and dynamic characteristics and various structures. Firstly, two new types of multi-degree-of-freedom and large-stroke compliant kinematic joints were designed. Then, three new types of spatial translational compliant micro-positioning stages with large motion range were designed based on these compliant kinematic joints. Next, Nonlinear method was used to modeling the force-displacement relationship, input coupling and lost motion of the stage, and compliance matrix method was used to modeling the stiffness and natural frequency of the stage. Afterwards, optimal parameter was searched to improve both static and dynamic performance of the stage. Finally, the validity of the theoretical model was proved by finite element simulation. According to the results of both theory and simulation, the first-order natural frequency of the stage is $49.6 \mathrm{~Hz}$. In $1 \mathrm{~mm}$ motion stroke, the lost motion in the $x$ and $z$ directions is less than $0.32 \%$ and $0.7 \%$, the input coupling is less than $1.2 \%$ and the output motion is completely decoupled. It provides a systematic research method for the design, analysis and optimization of spatial translational compliant micro-positioning stages.
\end{abstract}

Key words: compliant mechanisms; large-range; structure design; nonlinear modeling; parameter optimization

\section{0 前言}

柔性机构是现代机构学的重要分支, 它依靠材 料的变形来传递力或运动 ${ }^{11]}$, 由于机构中的运动副

* 江苏省 “六大人才高峰” 计划(ZBZZ-012)、高等学校学科创新引智计 划(B18027)和江苏省研究生创新计划(SJCX18-0630, KYCX18-1846) 资助项目。20191006 收到初稿, 20200114 收到修改稿
为一体化的结构, 故避免了间隙、摩擦、磨损、回 程误差，同时免于润滑、装配、频繁维护，利于实 现稳定的高精度运动。随着精密工程、纳米技术的 发展, 对微定位机构的功能要求从直线运动、二维

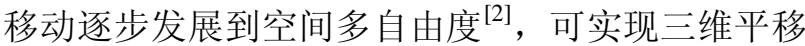
的柔性微定位平台在精密定位 ${ }^{[3]}$ 、微纳加工 ${ }^{[4]}$ 、精微

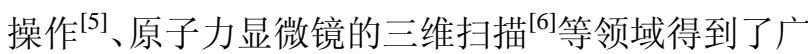
泛应用，国内外针对空间平动柔性机构的研究已取得 
了一定的成果, 李海洋等 ${ }^{[7-8]}$ 提出四杆和两杆型柔性运 动副, 并基于此设计了 3-PPPR、3-PPPRR 型分布柔 度式机构, 通过将自由度与约束空间拓扑法应用于空 间平动柔性机构的设计, 拓展了机构的多样性。TANG 等[9]采用大行程柔性移动副设计了 3-PPP 型机构, 由 于限制了移动副的寄生运动, 机构的耦合误差小于 $1.9 \%$ 。 LI 等 ${ }^{[10-11]}$ 提出具有多自由度的复合结构式柔 性运动副并设计了 3-RPC 型机构, 经过多步的结构 优化, 支链中的柔性铰链高度集成, 进而提高了机 构的静、动态性能与结构紧凑性。YUE 等 ${ }^{[12]}$ 根据

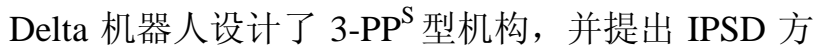
法展开系统化研究, 实现了对机构的高精度控制。 为提升机构的解耦性能并限制其伴生转动, LI 等 ${ }^{[13]}$ 和 ZHANG 等 ${ }^{[14]}$ 分别提出 6-PPPR、6-PPP 型支链对 称布置的空间平动柔性机构。

柔性机构在总体构型设计上与刚性并联机构可 相互借鉴, 而新型柔性运动副的设计与结构布局的优 化则是高性能、多样化柔性机构设计的关键。目前具 有大行程、良好静、动态特性且便于实现的高性能结 构设计与参数化尺寸设计实例仍较为圆乏, 因此从柔 性运动副的设计出发, 提出一种包含结构设计、静、 动态特性分析以及参数寻优的系统化研究方法。

\section{1 结构设计}

\section{1 大行程柔性运动副设计}

为了提升动态性能, 空间平动柔性微定位平台 一般采用驱动器可固定安装的并联式结构, 由于机 构具有三个移动自由度, 组成机构的支链均须包含 三个移动自由度, 因此含有移动自由度的大行程柔 性运动副是机构设计的关键。

图 1a 所示为具有两平移 (2 Translation)自由度 的柔性运动副, 采用两组片状梁单元通过交错布置 并反向串联而成, 末端设置有限制非柔性结构变形 的约束单元, 分布柔度式的结构可以保证运动副具 有较大的行程, 交错式的布局提高了结构的紧凑性, 反向串联的方法, 使得平台多轴联动时, 两组移动 副的寄生运动可相互抵消 $\left(e_{1}-e_{2}\right)$, 从而大幅降低平 台的耦合误差，其工作原理如图 2 所示。

图 $1 \mathrm{~b}$ 所示为具有两移一转 (2 Translation 1 Rotation)自由度的柔性运动副, 结构中包含 8 根柔 性细长杆和刚性连接模块, 该运动副是由两组四杆结 构反向串联而成的复合结构, 相较于单个四杆结构具 有更大的运动行程, 且变形时不产生寄生运动, 与已 有的结构 ${ }^{[15]}$ 相比布局紧凑、结构参数少、适应多种
加工方式、易于实现, 其工作原理如图 3 所示。

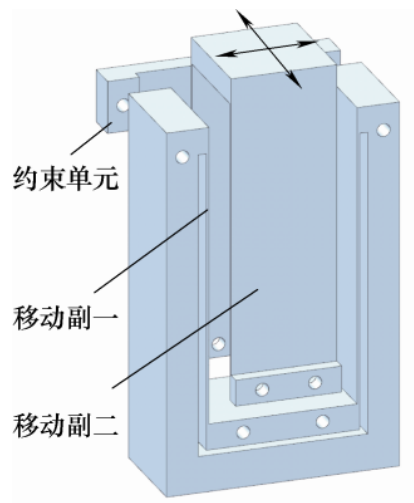

(a) $2 \mathrm{~T}$ 型柔性运动副

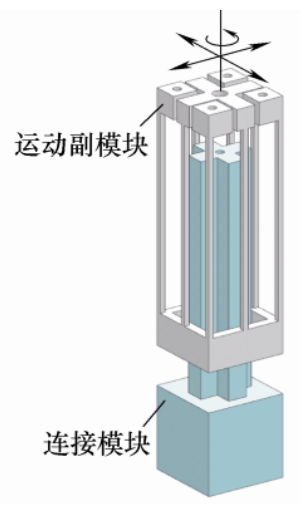

(b) 2T1R 型柔性运动副
图 1 两种大行程柔性运动副

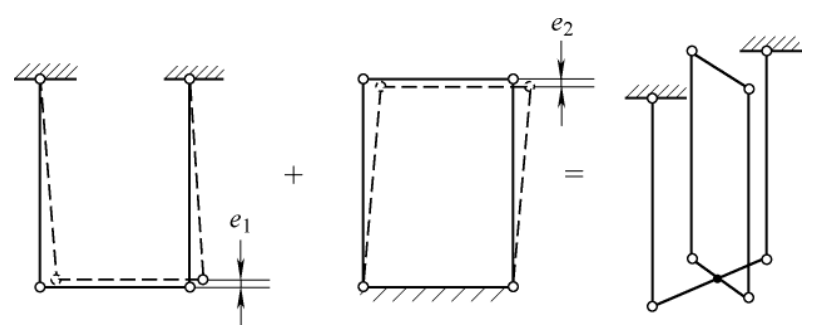

图 2 2 $\mathrm{T}$ 型柔性运动副工作原理

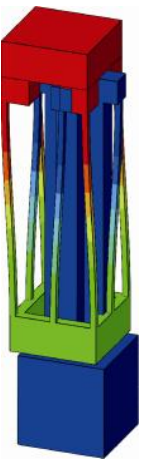

图 3 2T1R 型柔性运动副工作原理

\section{2 大行程三平动柔性微定位平台设计}

空间平动柔性微定位平台主要有三支链, 五支 $\mathrm{c}^{[16]}$, 六支链三种类型, 其中三支链平台结构简单, 构型种类较多, 但动平台受力不对称, 容易出现偏 转。六支链平台结构布局完全对称，可消除平台偏转 和由运动副寄生运动导致的平台输出耦合, 但结构复 杂, 常导致动平台有效台面减小。五支链平台兼具二 者的优点, 同时动平台有效台面不受结构布局的影响。

采用图 1a 中 $2 \mathrm{~T}$ 型柔性运动副设计了各向同性 3-PPP 型大行程柔性微定位平台如图 4 所示, 包括 3 条结构相同的柔性支链如图 5 所示, 其中两组对称 布置的片状梁单元作为支链的驱动副, $2 \mathrm{~T}$ 型柔性运 动副作为被动副。与现有的三支链平台相比结构紧 凑, 多轴驱动时不存在耦合误差累积现象。 


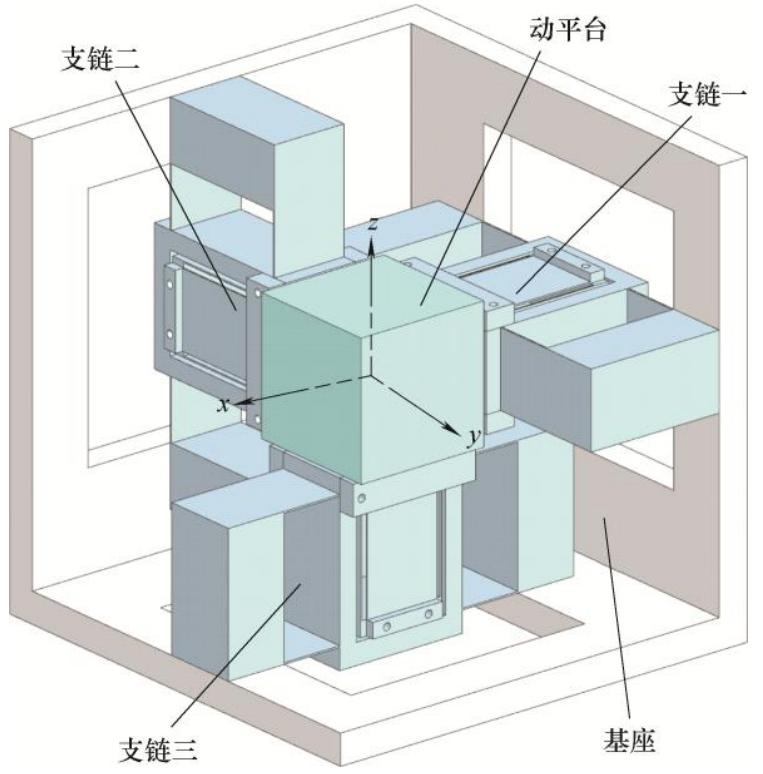

图 4 大行程微定位平台实例 1

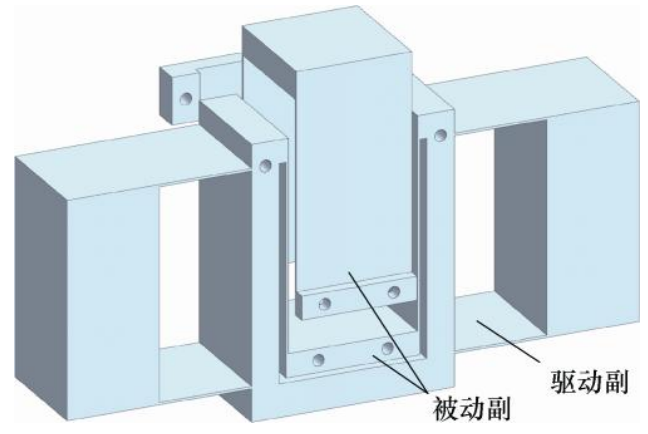

图 5 PPP 型柔性支链

采用图 $1 \mathrm{~b}$ 中 $2 \mathrm{~T} 1 \mathrm{R}$ 型柔性运动副设计了五支链大 行程柔性微定位平台如图 6 所示, $x 、 y$ 轴方向 4 条支 链相同如图 7a 所示, 包含 4 个 $2 \mathrm{~T} 1 \mathrm{R}$ 型柔性运动副, 其中 3 个并联构成支链的驱动副。 $z$ 轴方向支链如图 $7 b$ 所示, 包含 5 个 $2 \mathrm{~T} 1 \mathrm{R}$ 型柔性运动副, 其中 4 个并联构 成支链的驱动副。由于机构中所有的运动副均为同一 模块的大行程运动副, 且变形时不产生寄生运动, 故 平台运动行程大、尺寸参数少、输入输出完全解耦。

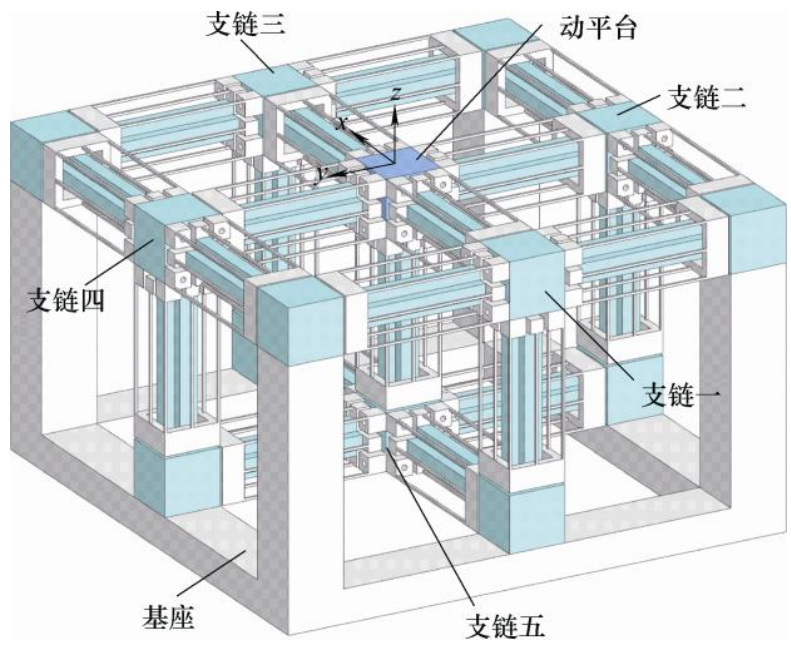

图 6 大行程微定位平台实例 2

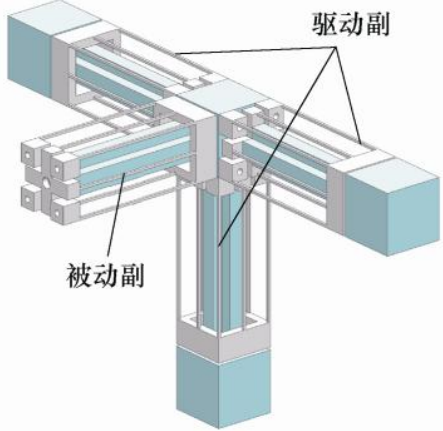

(a) 支链二结构

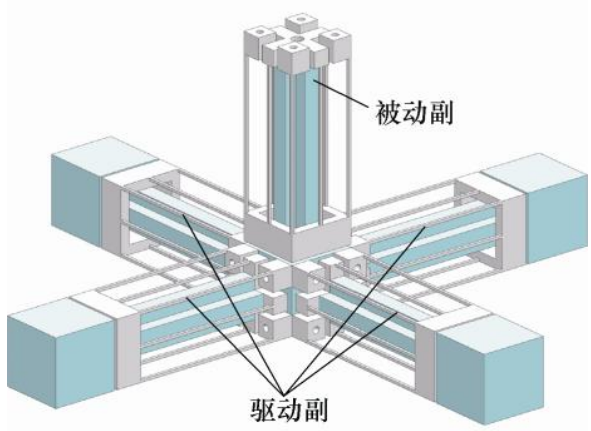

图 7 平台实例 2 支链结构

同时采用图 $1 \mathrm{a} 、 1 \mathrm{~b}$ 柔性运动副设计了五支链大 行程柔性微定位平台如图 8 所示, 平台在 $x 、 y$ 轴方 向包含 4 条与图 5 结构相同的支链, 并作对称布置, 以消除动平台在 $x 、 y$ 方向的输出耦合， $z$ 轴方向支 链如图 9 所示, 驱动副由四杆结构运动副并联而成, 以提升结构刚度, 被动副为无寄生运动的 $2 \mathrm{~T} 1 \mathrm{R}$ 型 柔性运动副, 以消除平台在 $z$ 轴方向的输入、输出 耦合。五支链的布局、分布柔度式的柔性单元、以 及运动副的复合结构, 使得平台结构紧凑、运动行 程大、有效台面大、输出完全解耦。

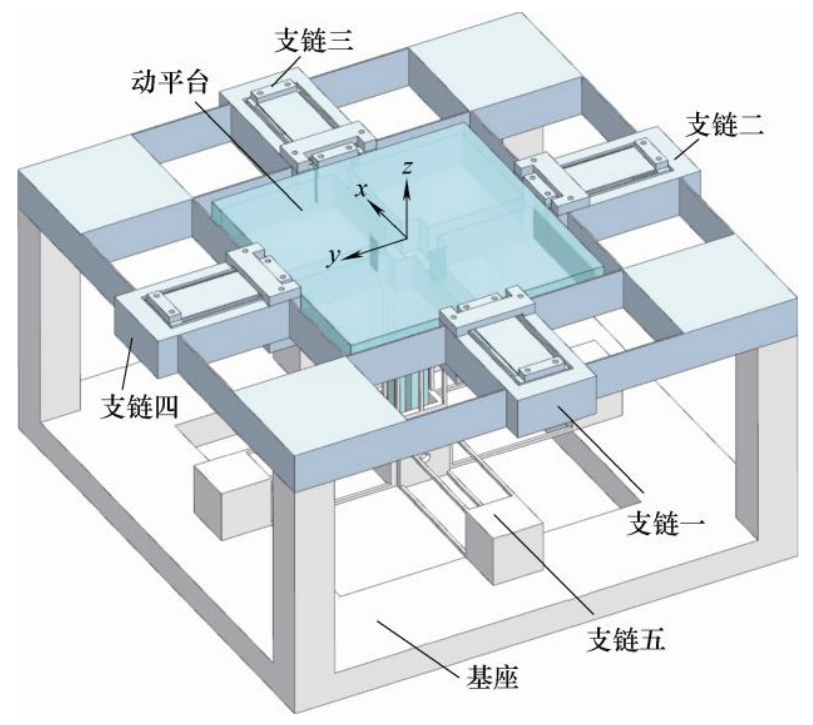

图 8 大行程微定位平台实例 3 


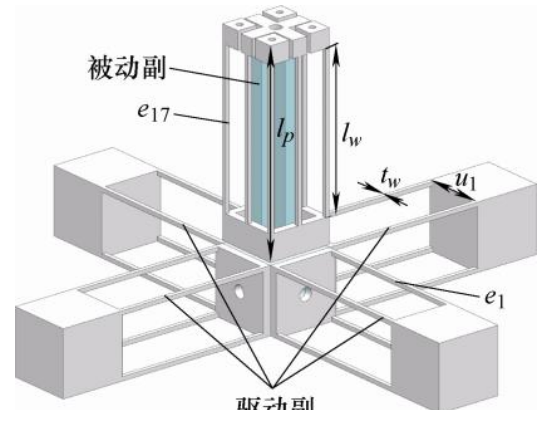

图 $9 z$ 轴方向支链

在上述 3 个设计实例的动平台中心处建立总体 坐标系, 根据螺旋理论, 并联机构的自由度为各支 链自由度的交集, 因此平台设计实例 $1 \sim 3$ 的自由度 可进一步验证为

$$
\begin{aligned}
&\{x, y, z\}_{1} \cap\{x, y, z\}_{2} \cap\{x, y, z\}_{3}=\{x, y, z\} \\
&\left\{x, y, z, \theta_{x}\right\}_{1} \cap\left\{x, y, z, \theta_{y}\right\}_{2} \cap\left\{x, y, z, \theta_{x}\right\}_{3} \cap \\
&\left\{x, y, z, \theta_{y}\right\}_{4} \cap\left\{x, y, z, \theta_{z}\right\}_{5}=\{x, y, z\} \\
&\{x, y, z\}_{1} \cap\{x, y, z\}_{2} \cap\{x, y, z\}_{3} \cap\{x, y, z\}_{4} \cap \\
&\left\{x, y, z, \theta_{z}\right\}_{5}=\{x, y, z\}
\end{aligned}
$$

将上述大行程柔性运动副与现有的柔性运动副相 结合, 或对现有的微定位平台进行运动副替换, 还可 得到多种新型的大行程微定位平台, 不再一一列举。

\section{2 静、动态特性分析}

以图 8 平台为例, 进行静、动态特性分析。目前 的分析方法有柔度矩阵法 ${ }^{[17-18]}$ 、伪刚体模型法 ${ }^{[16]}$ 、梁 约束模型法 ${ }^{[19-20]}$ 以及简化的非线性模型法 ${ }^{[21]}$, 根据平 台的结构特点并综合考虑模型精度和简便性, 将简化 的非线性模型法进行一定的修正和完善对平台进行静 态特性分析, 采用柔度矩阵法进行动态特性分析。

\section{$2.1 x 、 y$ 轴方向的力-位移关系分析}

由平台结构的对称性可知, 在 $x$ 和 $y$ 轴方向平 台具有相同的静、动态特性, 因此以 $x$ 轴方向为例, 建立平台在 $x 、 y$ 轴方向的力-位移关系模型。

如图 10 所示, 当沿 $x$ 轴方向施加单轴驱动位移 $\delta_{x}$ 时, 支链一、三中的驱动副, 支链二、四、五中的被 动副产生弹性变形, 通过建立单根梁单元的力-位移关 系模型，可得到平台整体在 $x$ 轴方向的力-位移关系， 平台中片状梁、细长杆均具有相同的尺寸参数。

支链一驱动副中梁 $a_{1}$ 的力-位移关系模型如图 11 所示, 梁 $a_{1}$ 产生弯曲和拉伸变形且在 $y$ 轴方向的投影 长度保持不变, 根据点 $A_{1}$ 在 $x$ 轴方向的受力平衡条 件, 驱动力 $F_{a_{1} x}$ 与驱动位移 $\delta_{x}$ 之间的关系为

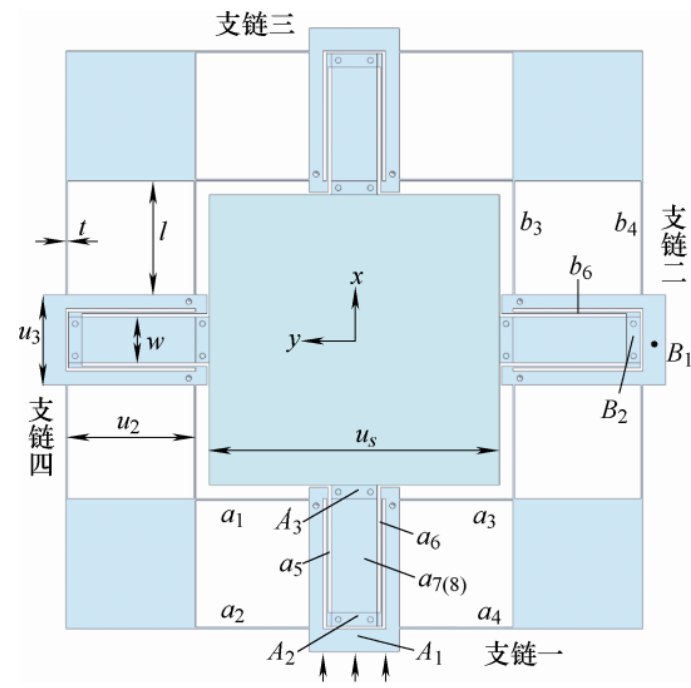

图 10 平台在 $X Y$ 平面内的结构

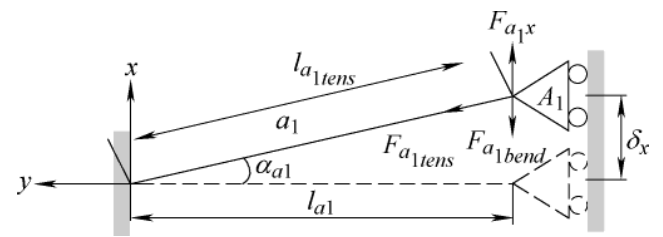

图 11 梁 $a_{1}$ 的力-位移关系模型

$$
\begin{gathered}
F_{a_{1} x}=F_{a_{1} \text { bend }}+F_{a_{1} \text { tens }} \sin \alpha_{a_{1}} \\
F_{a_{1} \text { bend }}=\frac{E^{\prime} t^{3} w}{l_{a_{1}}^{3}} \delta_{x} \\
F_{a_{1} \text { tens }}=\frac{E^{\prime} t w}{l_{a_{1}}}\left(l_{a_{1} \text { tens }}-l_{a_{1}}\right) \\
l_{a_{1} \text { tens }}=\sqrt{l_{a_{1}}^{2}+\delta_{x}^{2}} \\
\sin \alpha_{a_{1}}=\frac{\delta_{x}}{l_{a_{1} \text { tens }}}
\end{gathered}
$$

式中, $F_{a_{1} \text { bend }} 、 F_{a_{1} \text { tens }}$ 分别为弯曲、拉伸变形作用力; $\alpha_{a_{1}}$ 为变形前后的夹角; $l_{a_{1}}$ 为梁 $a_{1}$ 的原长; $l_{a_{1} \text { tens }}$ 为 梁 $a_{1}$ 变形后的长度; $t$ 为梁的厚度; $w$ 为梁的宽度, 对于片状梁单元当 $w>10 t$ 时, 应将弹性模量 $E$ 修 正为平面模量 $E^{\prime}$ 二者之间的关系为 ${ }^{[22]}$

$$
E^{\prime}=\frac{E}{1-v^{2}}
$$

式中, $v$ 为材料的泊松比。

支链二被动副中梁 $b_{6}$ 的力-位移关系模型如图 12 所示, 梁 $b_{6}$ 变形时, 根据其两端点的相对运动, 可等 效为固定导向梁模型。根据 HOWELL 提出的固定导 向梁 $2 R$ 伪刚体模型 ${ }^{[1]}$ 对简化的非线性模型进行修 正，其在 $y$ 轴方向的投影长度为 


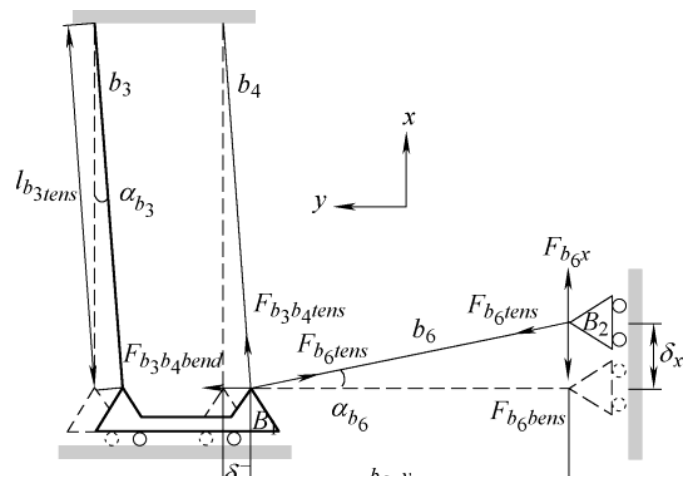

图 12 梁 $b_{6}$ 的力-位移关系模型

$$
l_{b_{6}-y}=l_{b_{6}}-0.8517 l_{b_{6}}\left(1-\sqrt{1-\left(\frac{\delta_{x}}{0.8517 l_{b_{6}}}\right)^{2}}\right)
$$

根据点 $B_{2}$ 在 $x$ 轴方向的受力平衡条件, 驱动力 $F_{b_{6} x}$ 与驱动位移 $\delta_{x}$ 之间的关系为

$$
\begin{gathered}
F_{b_{6} x}=F_{b_{6} \text { tens }} \sin \alpha_{b_{6}}+F_{b_{6} \text { bend }} \\
\sin \alpha_{b_{6}}=\frac{\delta_{x}}{\sqrt{\left(l_{b_{6}-y}\right)^{2}+\left(\delta_{x}\right)^{2}}} \\
F_{b_{6} \text { bend }}=\frac{E^{\prime} t^{3} w}{l_{b_{6}}^{3}} \delta_{x}
\end{gathered}
$$

根据点 $B_{1}$ 在 $y$ 轴方向的受力平衡条件可得

$$
\begin{gathered}
F_{b_{6} \text { tens }}=\frac{F_{b_{3} b_{4} \text { bend }}+F_{b_{3} b_{4} \text { tens }} \sin \alpha_{b_{3}}}{\cos \alpha_{b_{6}}} \\
\cos \alpha_{b_{6}}=\frac{l_{b_{6}-y}}{\sqrt{\left(l_{b_{6}-y}\right)^{2}+\left(\delta_{x}\right)^{2}}} \\
F_{b_{3} b_{4} b \text { end }}=2 \frac{E^{\prime} t^{3} w}{l_{b_{3}}^{3}} \delta_{y}^{-} \\
\delta_{y}^{-}=l_{b_{6}}-l_{b_{6}-y} \\
F_{b_{3} b_{4} \text { tens }}=2 \frac{E^{\prime} t w}{l_{b_{3}}}\left(l_{b_{3} \text { tens }}-l_{b_{3}}\right) \\
l_{b_{3} \text { tens }}=\sqrt{l_{b_{3}}^{2}+\left(\delta_{y}^{-}\right)^{2}} \\
\sin \alpha_{b_{3}}=\frac{\delta_{y}^{-}}{l_{b_{3} \text { tens }}}
\end{gathered}
$$

式中, $F_{b_{6} \text { bend }} 、 F_{b_{6} \text { tens }}$ 分别为梁 $b_{6}$ 弯曲、拉伸变形作 用力; $F_{b_{3} b_{4} \text { bend }} 、 F_{b_{3} b_{4} \text { tens }}$ 分别为梁 $b_{3}$ 和 $b_{4}$ 弯曲、拉伸 变形合力; $l_{b_{3}} 、 l_{b_{6}}$ 分别为梁 $b_{3} 、 b_{6}$ 的原长; $l_{b_{3} \text { tens }}$ 为 梁 $b_{3}$ 拉伸变形后的长度; $\alpha_{b_{3}} 、 \alpha_{b_{6}}$ 分别为梁 $b_{3} 、 b_{6}$ 变形前后的夹角。

由图 12 可知当沿 $x$ 轴方向施加驱动位移 $\delta_{x}$ 时, 梁 $b_{6}$ 所在被动副产生 $y$ 轴方向的寄生运动, 导致支 链二输入点处产生沿 $y$ 轴负方向(背离动平台中心方
向)的耦合位移 $\delta_{y}^{-}$, 这种由不同输入之间相互干扰所 造成的位移称为平台的输入耦合, 动平台在非输入方 向上的位移称为平台的输出耦合, 为保证平台具有良 好的静态特性, 应减小平台的输入、输出耦合。

支链五中的 $2 \mathrm{~T} 1 \mathrm{R}$ 型被动副由两组四杆结构反 向串联而成, 变形时无应力刚化效应, 故其力-位移 关系是线性的，每组四杆结构的刚度为

$$
k_{f b}=4 \frac{E t_{w}^{4}}{l_{w}^{3}}
$$

则该被动副的刚度及力位移关系为

$$
\begin{gathered}
k_{d f b}=\frac{k_{f b}}{2}=2 \frac{E t_{w}^{4}}{l_{w}^{3}} \\
F_{d f b x}=k_{d f b} \delta_{x}
\end{gathered}
$$

式中, $l_{w} 、 t_{w}$ 分别为细长杆的长度、厚度。

根据式(4)、(11)、(23)在 $x$ 轴方向施加驱动位移 $\delta_{x}$ 所需驱动力为

$$
F_{x}=8 F_{a_{1} x}+4 F_{b_{6} x}+F_{d f b x}
$$

\section{$2.2 z$ 轴方向的力-位移关系分析}

当沿 $z$ 轴方向施加单轴驱动位移 $\delta_{z}$ 时, 支链五 中的驱动副、支链一至四中的被动副产生弹性变形。

支链五驱动副中细长杆 $e_{1}$ 的驱动力 $F_{e_{1} z}$ 与驱动 位移 $\delta$ 之间的关系模型与图 11 相同, 不再详述。 支链一被动副中梁 $a_{7}$ 的力-位移关系模型如图 13 所 示, 根据点 $A_{3}$ 在 $z$ 轴方向的受力平衡条件, 驱动力 $F_{a_{7} z}$ 与驱动位移 $\delta_{z}$ 之间的关系为

$$
\begin{gathered}
F_{a_{7} z}=F_{a_{7} \text { bend }}+F_{a_{7} \text { tens }} \sin \alpha_{a_{7}} \\
F_{a_{7} \text { bend }}=\frac{E^{\prime} t^{3} w}{l_{a_{7}}^{3}} \delta_{z} \\
F_{a_{7} \text { tens }}=\frac{F_{a_{7} \text { tens }-x}}{\cos \alpha_{a_{7}}}
\end{gathered}
$$

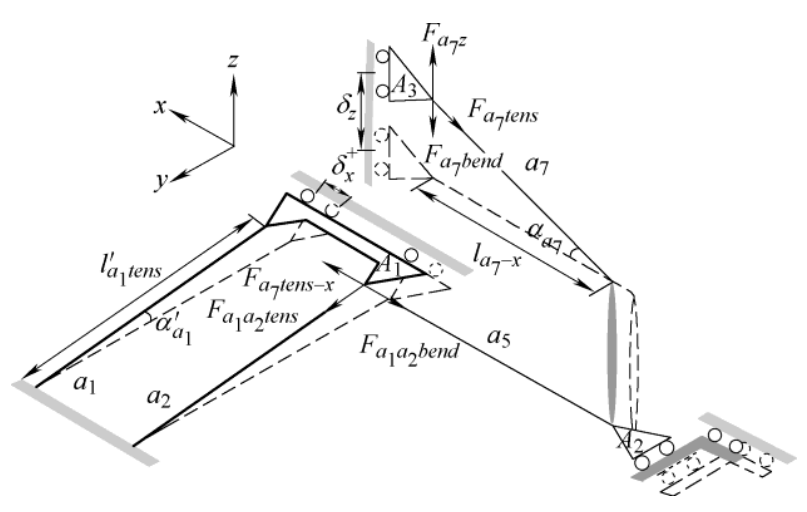

图 13 梁 $a_{7}$ 的力-位移关系模型

$$
\sin \alpha_{a_{7}}=\frac{\delta_{z}}{\sqrt{\left(l_{a_{7}-x}\right)^{2}+\left(\delta_{z}\right)^{2}}}
$$




$$
\begin{gathered}
\cos \alpha_{a_{7}}=\frac{l_{a_{7}-x}}{\sqrt{\left(l_{a_{7}-x}\right)^{2}+\left(\delta_{z}\right)^{2}}} \\
l_{a_{7}-x}=l_{a_{7}}-0.8517 l_{a_{7}}\left(1-\sqrt{1-\left(\frac{\delta_{z}}{0.8517 l_{a_{7}}}\right)^{2}}\right)
\end{gathered}
$$

根据点 $A_{1}$ 在 $x$ 轴方向的受力平衡条件可得

$$
\begin{gathered}
F_{a_{1} \text { tens }-x}=F_{a_{1} a_{2} \text { bend }}-F_{a_{1} a_{2} \text { tens }} \sin \alpha_{a_{1}}^{\prime} \\
F_{a_{1} a_{2} \text { bend }}=2 \frac{E^{\prime} t^{3} w}{l_{a_{1}}^{3}} \delta_{x}^{+} \\
F_{a_{1} a_{2} \text { tens }}=2 \frac{E^{\prime} A}{l_{a_{1}}}\left(l_{a_{1} \text { tens }}^{\prime}-l_{a_{1}}\right) \\
\sin \alpha_{a_{1}}^{\prime}=\frac{\delta_{x}^{+}}{l_{a_{1} \text { tens }}^{\prime}} \\
\delta_{x}^{+}=l_{a_{7}}-l_{a_{1_{1}-x}} \\
l_{a_{1} \text { tens }}^{\prime}=\sqrt{l_{a_{1}}^{2}+\left(\delta_{x}^{+}\right)^{2}}
\end{gathered}
$$

式中, $F_{a_{7} \text { bend }} 、 F_{a_{7} \text { tens }}$ 为梁 $a_{7}$ 弯曲、拉伸变形作用力, $F_{a_{7} \text { tens }-x}$ 为 $F_{a_{7} \text { tens }}$ 在 $x$ 轴方向的分力; $F_{a_{1} a_{2} b e n d} 、 F_{a_{1} a_{2} \text { tens }}$ 分别为梁 $a_{1}$ 和 $a_{2}$ 的弯曲、拉伸变形合力; $l_{a_{7}}$ 为梁 $a_{7}$ 的长度; $l_{a_{7}-x}$ 为梁 $a_{7}$ 在 $x$ 轴方向的投影长度; $l_{a_{1} \text { tens }}^{\prime}$ 为 梁 $a_{1}$ 拉伸变形后的长度; $\alpha_{a_{1}}^{\prime} 、 \alpha_{a_{7}}$ 分别为梁 $a_{1} 、 a_{7}$ 变形前后的夹角。

由图 13 可知当沿 $z$ 轴方向施加驱动位移 $\delta$ 时, 梁 $a_{7}$ 所在被动副产生 $x$ 轴方向的寄生运动, 导致支 链一输入点处产生沿 $x$ 轴负方向(指向动平台中心方 向)的耦合位移 $\delta_{x}^{+}$, 同时支链二至支链四的输入点 均会产生指向动平台中心方向且数值为 $\delta_{x}^{+}$的耦合 位移。根据式(10)、(17)、(30)、(35)可知, 当沿 $x$ 轴和 $z$ 轴驱动位移相等时, 在 $y$ 轴输入点处产生的 耦合位移大小相等方向相反，相互抵消。

根据发生变形的运动副中单根梁单元的力-位 移关系模型, 同时考虑重力作用, 在 $z$ 轴方向施加 驱动位移 $\delta_{z}$ 所需驱动力为

$$
F_{z}=16 F_{e_{1} z}+8 F_{a_{7} z}+\left(\frac{1}{2} M_{1}+M_{2}\right) g
$$

式中, $M_{1}$ 为发生弹性变形的梁单元质量之和; $M_{2}$ 为在 $z$ 轴方向平动的刚、柔性部件质量之和。

\section{3 丢失运动分析}

丢失运动是指平台输入、输出位移之间的差值, 柔性单元在其非自由度方向受力时仍会产生微小变 形, 导致驱动器的运动不能完全传递到动平台, 从 而降低了系统的定位精度, 为保证平台具有良好的 静态特性并简化控制系统, 应减小平台的丢失运动。

造成实例 3 平台丢失运动的主要因素是, 驱动
力作用的支链中被动副的拉伸、压缩变形。重力是 恒力, 在无输入与输出时就已经作用于平台, 驱动 时不会额外造成平台输入、输出间的差值。因此, 基于上述力-位移关系分析，并结合胡克定律， $x 、 z$ 轴方向的丢失运动 $\delta_{\text {xlost }} 、 \delta_{\text {zlost }}$ 为

$$
\begin{gathered}
\delta_{x l o s t}=\frac{\left(F_{x}-4 F_{a_{1} x}\right) l}{E^{\prime} t w} \\
\delta_{z l o s t}=\frac{4 F_{a_{7} z} l_{w}}{E t_{w}^{2}}
\end{gathered}
$$

式中, $l$ 为片状梁的长度。

\section{4 材料应力分析}

为保证材料的变形在弹性范围内需要进行应力 分析, 平台工作时驱动副中的梁单元同时承受弯曲 正应力和较大的拉伸应力最易发生材料失效, 其中 片状梁末端的最大应力为

$$
\begin{gathered}
\sigma_{\max 1}=\sigma_{a_{1} \text { bend }}+\sigma_{a_{1} \text { tens }} \\
\sigma_{a_{1} \text { bend }}=\frac{3 E^{\prime} t \delta_{x}}{l^{2}} \\
\sigma_{a_{1} \text { tens }}=\varepsilon_{a_{1}} E^{\prime} \\
\varepsilon_{a_{1}}=\frac{l_{a_{1} \text { tens }}-l}{l}
\end{gathered}
$$

细长杆末端最大应力为

$$
\begin{gathered}
\sigma_{\max 2}=\sigma_{e_{1} \text { bend }}+\sigma_{e_{1} \text { tens }} \\
\sigma_{e_{1} \text { bend }}=\frac{3 E t_{w} \delta_{z}}{l_{w}^{2}} \\
\sigma_{e_{1} \text { tens }}=\varepsilon_{e_{1}} E \\
\varepsilon_{e_{1}}=\frac{l_{e_{1} \text { tens }}-l_{w}}{l_{w}}
\end{gathered}
$$

式中, $\sigma_{a_{1} \text { bend }} 、 \sigma_{a_{1} \text { tens }}$ 分别为梁 $a_{1}$ 的弯曲、拉伸应力; $\varepsilon_{a_{1}}$ 为梁 $a_{1}$ 的应变; $\sigma_{e_{1} \text { bend }} 、 \sigma_{e_{1} \text { tens }}$ 分别为细长杆的 弯曲、拉伸应力; $\varepsilon_{e_{1}} 、 l_{e_{1} \text { tens }}$ 分别为梁 $e_{1}$ 的应变、变 形后的长度。

\section{5 屈曲分析}

对于分布柔度式平台, 在材料屈服失效之前, 承受压力的梁单元更易先发生屈曲或失稳现象, 实 例 3 平台中 $2 \mathrm{~T}$ 型运动副及 $2 \mathrm{~T} 1 \mathrm{R}$ 型运动副均是反向 串联结构, 工作时倒置的梁单元承受拉力故无屈曲 风险, 非倒置部分承受压力, 选取其中片状梁 $a_{7}$ 、 细长杆 $e_{17}$ 进行屈曲分析。基于上述平台的力-位移 关系模型， $a_{7} 、 e_{17}$ 承受的压力 $F_{p 1} 、 F_{p 2}$ 分别为 


$$
\begin{gathered}
F_{p 1}=\frac{1}{2}\left(F_{x}-4 F_{a_{1} x}\right) \\
F_{p 2}=\frac{1}{4}\left(8 F_{a_{7} z}+F_{\text {load }}\right)+\left(\frac{1}{2} M_{1}+M_{2}\right) g
\end{gathered}
$$

根据欧拉公式, $a_{7} 、 e_{17}$ 可承受的临界压力 $F_{c r 1}$ 、 $F_{c r 2}$ 分别为

$$
\begin{aligned}
& F_{c r 1}=\frac{\pi^{2} E^{\prime} I_{1}}{\left(\frac{l}{2}\right)^{2}} \\
& F_{c r 2}=\frac{\pi^{2} E I_{2}}{\left(\frac{l_{w}}{2}\right)^{2}}
\end{aligned}
$$

式中, $F_{\text {load }}$ 为外部载荷, $I_{1} 、 I_{2}$ 分别为梁 $a_{7} 、 e_{17}$ 的 截面惯性矩。

\section{6 模态分析}

模态是结构的固有振动特性, 属于平台的动态 特性, 为了增加工作带宽, 避免在高频驱动时出现 共振现象, 需要进行模态分析以提升固有频率, 平 台的前三阶固有频率为

$$
\begin{aligned}
f & =\sqrt{k_{\text {input }} / M_{\text {eq }}} / 2 \pi \\
M_{\text {eq }} & =\frac{13}{35} M_{\text {deform }}+M_{\text {move }}
\end{aligned}
$$

式中, $M_{e q}$ 为平台在各移动自由度方向的等效质量 ${ }^{[22]}$, $M_{\text {deform }} 、 M_{\text {move }}$ 分别为各轴方向, 发生弹性变形的梁 单元质量之和以及平动的刚、柔性部件质量之和。

$k_{\text {input }}$ 为各轴方向的输入刚度, 基于柔度矩阵法的平 台刚度分析实例参阅文献[17-18], 不再详述。

\section{3 参数寻优}

为同时提高平台的静、动态性能需要对平台 进行参数寻优, 选择密度低、强度与弹性模量比 高的 AL-7075 作为平台材料, 其弹性模量 $E$ 为 $71700 \mathrm{MPa}$, 泊松比 $v$ 为 0.33 , 屈服强度 $\sigma$ 为 $503 \mathrm{MPa}$, 密度 $2810 \mathrm{~kg} / \mathrm{m}^{3}$ 。

利用 Matlab 中的 fmincon 和 fgoalattain 函数, 采用目标规划法进行参数寻优, 由上述平台结构与 理论分析可知柔性梁单元的尺寸参数是平台具体尺 寸与静、动态性能的决定性因素, 考虑制造的经济 性, 设定片状梁宽度 $w$ 为整数值 $20 \mathrm{~mm}$, 因此设计 变量为

$$
\boldsymbol{X}=\left[l, t, l_{w}, t_{w}\right]^{\mathrm{T}}
$$

设计变量的变化范围为

$$
\left\{\begin{array}{l}
\boldsymbol{X}_{\mathrm{L}}=[20,0.3,20,0.3]^{\mathrm{T}} \\
\boldsymbol{X}_{\mathrm{U}}=[60,2,60,2]^{\mathrm{T}}
\end{array}\right.
$$

为了提升平台的静、动态性能, 需要增大平台 的固有频率, 同时减小输入耦合与丢失运动, 因此 目标函数为

$$
\left\{\begin{array}{l}
f_{1}(\boldsymbol{X})=-f_{x} \\
f_{2}(\boldsymbol{X})=-f_{z} \\
f_{3}(\boldsymbol{X})=\delta_{x l o s t} \\
f_{4}(\boldsymbol{X})=\delta_{z l o s t} \\
f_{5}(\boldsymbol{X})=\delta_{x}^{+} \\
f_{6}(\boldsymbol{X})=\delta_{y}^{-}
\end{array}\right.
$$

平台至少应有 $1 \mathrm{~mm}$ 的运动行程、 $50 \mathrm{~Hz}$ 固有 频率，平台最大应力不应超过许用应力，且不应发 生屈曲现象。设定平台承载 $F_{\text {load }}$ 为 $20 \mathrm{~N}$, 为方便大 行程驱动器音圈电机选型, 设定平台所需驱动力不 超过 $100 \mathrm{~N}$, 因此约束条件为

$$
\begin{gathered}
\delta_{x}=1 \quad \delta_{z}=1 \quad w=20 \\
\sigma_{\max 1} \leqslant \frac{\sigma}{k} \quad \sigma_{\mathrm{max}} \lesssim \frac{\sigma}{k} \\
F_{p 1} \leqslant \frac{F_{c r 1}}{k} \quad F_{p 2} \leqslant \frac{F_{c r 2}}{k} \\
F_{x} \leqslant 100 \quad F_{z}+F_{\text {load }} \leqslant 100 \\
f_{x} \geqslant 50 \quad f_{z} \geqslant 50
\end{gathered}
$$

式中, $f_{x} 、 f_{z}$ 分别为 $x 、 z$ 轴方向的固有频率, 为 保证可靠性设定安全系数 $k=2$ 。

参数寻优后平台的体积为 $272.22 \times 272.22 \times$ $143 \mathrm{~mm}^{3}$, 主要尺寸参数及各项静、动态性能如 表 1、2 所示。基于上述理论模型, 实现了平台的 参数化设计, 可大大提高不同性能参数要求下平 台的设计效率。

表 1 平台的尺寸参数 $(\mathrm{mm})$

\begin{tabular}{cc||cc}
\hline 参数 & 数值 & 参数 & 数值 \\
\hline$l$ & 49.7 & $l_{p}$ & 77 \\
$t$ & 0.71 & $u_{1}$ & 25 \\
$w$ & 20 & $u_{2}$ & 56.7 \\
$l_{w}$ & 60 & $u_{3}$ & 39.42 \\
$t_{w}$ & 1.56 & $u_{s}$ & 126.82 \\
\hline
\end{tabular}

表 2 平台的静、动态性能参数

\begin{tabular}{cc}
\hline 参数 & 数值 \\
\hline 固有频率 $f_{x} / \mathrm{Hz}$ & 50.22 \\
固有频率 $f_{z} / \mathrm{Hz}$ & 59.73 \\
最大输入耦合 $\delta_{x}^{+} / \mu \mathrm{m}$ & 11.81 \\
\hline
\end{tabular}


最大输入耦合 $\delta_{y}^{-} / \mu \mathrm{m}$

最大丢失运动 $\delta_{\text {xlost }} / \mu \mathrm{m}$

11.81

最大丢失运动 $\delta_{\text {zlost }} / \mu \mathrm{m}$

6.46

\section{4 仿真验证}

为验证理论模型的正确性, 采用 ABAQUS 对平台进行有限元仿真, 选择四面体单元进行网 格划分, 柔性单元处设置较小的网格划分参数(取 $1.2 \mathrm{~mm}$ ), 其余部分网格尺寸为 $5 \mathrm{~mm}$, 以提高结 果准确性和分析速度, 在平台与基座接触的面上 施加固定约束。

\section{$4.1 x$ 轴方向静态特性验证}

如图 14 所示, 沿 $x$ 轴方向施加 $0 \sim 1 \mathrm{~mm}$ 的驱 动位移, 平台各项静态性能的理论、仿真值如图 15 所示。

图 15a 所示为反作用力 $F_{x}$ 的理论(式(24))、仿真 值曲线, 最大值分别为 $97.46 \mathrm{~N} 、 98.30 \mathrm{~N}$, 在 0 $1 \mathrm{~mm}$ 驱动位移内, 理论与仿真之间的误差为 $0.2 \% \sim 6.5 \%$ 。

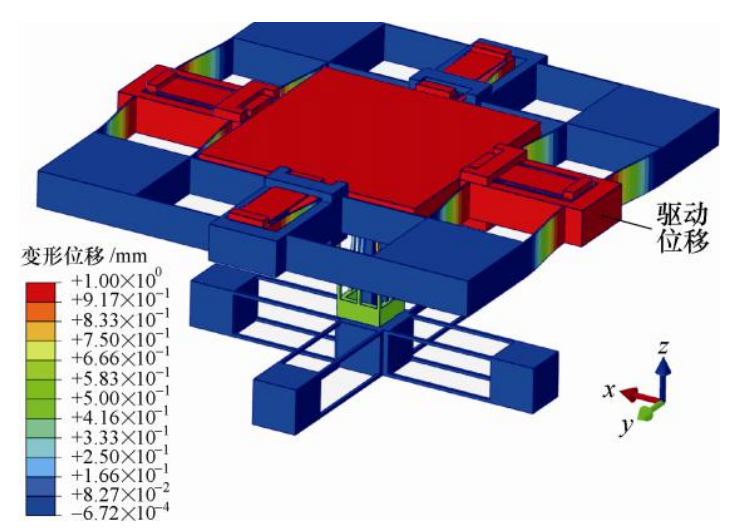

图 $14 x$ 轴方向的有限元仿真

图 $15 \mathrm{~b}$ 所示为 $y$ 轴输入点处耦合位移 $\delta_{y}^{-}$的理 论(式(17))、仿真值曲线, 极值分别为 $-11.81 \mu \mathrm{m}$ 、 $-11.92 \mu \mathrm{m}$, 小于驱动位移的 $1.2 \%$ 。在 $0 \sim 1 \mathrm{~mm}$ 驱动位移内, 理论与仿真之间的误差为 $0.9 \%$ $8.4 \%$ 。

图 15c 所示为丢失运动 $\delta_{x l o s t}$ 的理论(式(38))、仿 真值曲线, 最大值分别为 $2.61 \mu \mathrm{m} 、 3.11 \mu \mathrm{m}$, 小于 驱动位移的 $0.32 \%$ 。在 $0 \sim 1 \mathrm{~mm}$ 驱动位移内, 理论 与仿真之间的误差为 $1.5 \% \sim 19.1 \%$, 随着驱动位移 的增加, 被视为刚性部件的微小变形对仿真结果产 生了一定影响, 但丢失运动仿真值并未发生明显突 变, 表明片状梁无屈曲现象。

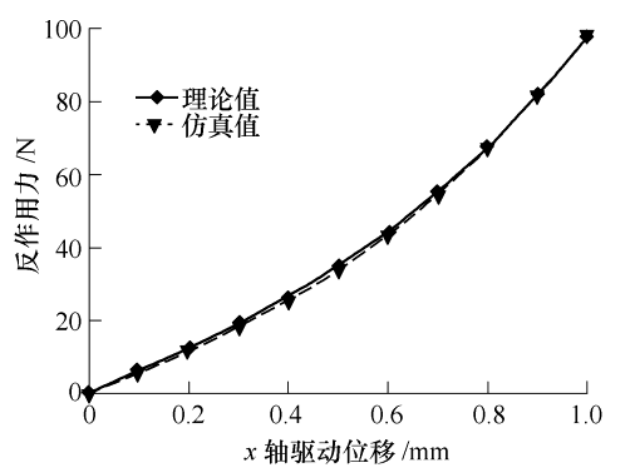

(a) $x$ 轴方向的反作用力

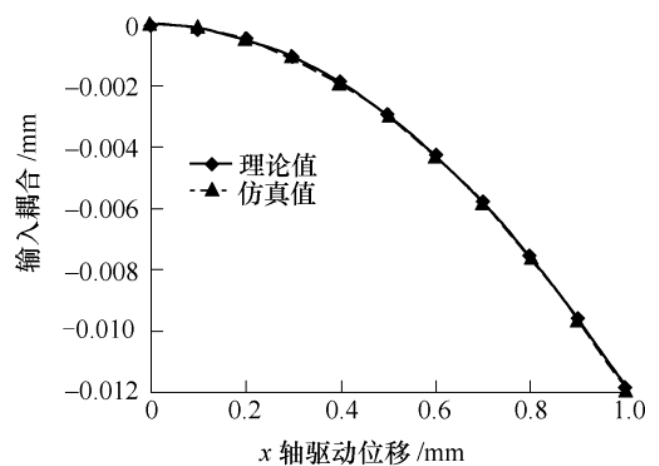

(b) $y$ 轴输入点的耦合位移

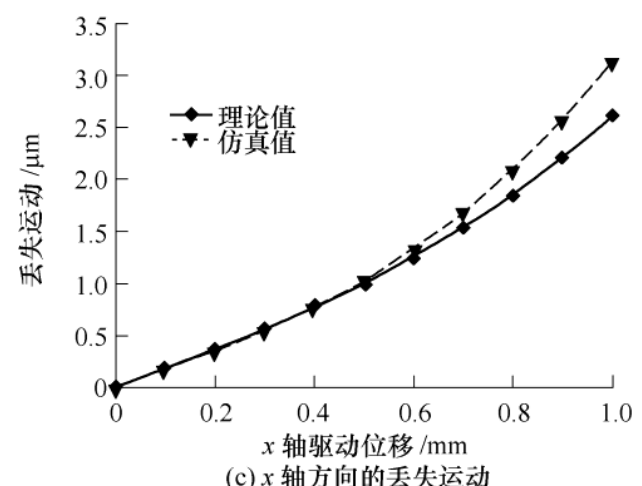

图 $15 x$ 轴方向平台静态性能验证

根据仿真结果, 结构中最大应力为 $109 \mathrm{MPa}$ 小 于材料许用应力。动平台中心点在 $y 、 z$ 轴方向的耦 合位移及 $z$ 轴方向输入点的耦合位移均小于 $0.1 \mu \mathrm{m}$, 其值完全可忽略不计, 表明 $x$ 轴驱动时, 平台输出、 $z$ 轴输入完全解耦。

\section{$4.2 z$ 轴方向静态特性验证}

如图 16 所示, 沿 $z$ 轴方向施加 $0 \sim 1 \mathrm{~mm}$ 的驱 动位移, 重力加速度 $g=10$ 沿 $z$ 轴负方向, 平台各项 静态性能的理论、仿真值如图 17 所示。 


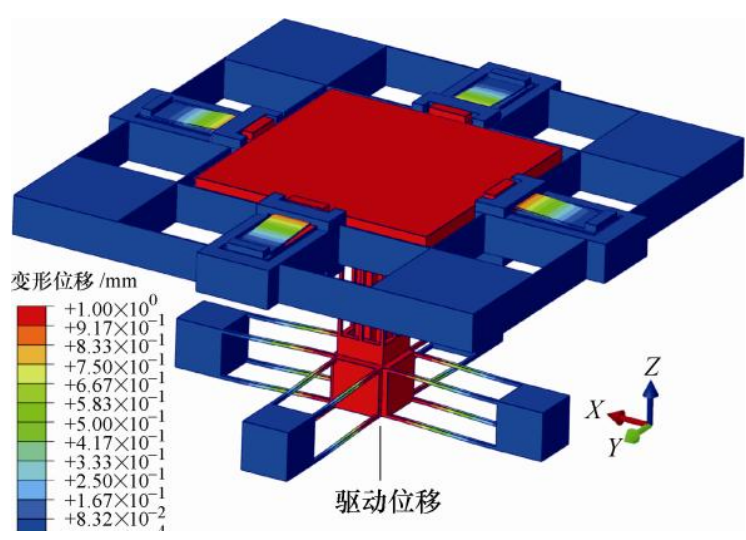

图 $16 z$ 轴方向的有限元仿真

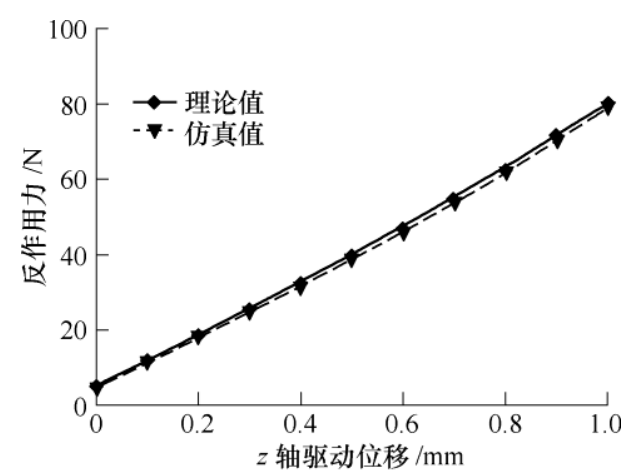

(a) $z$ 轴方向的反作用力

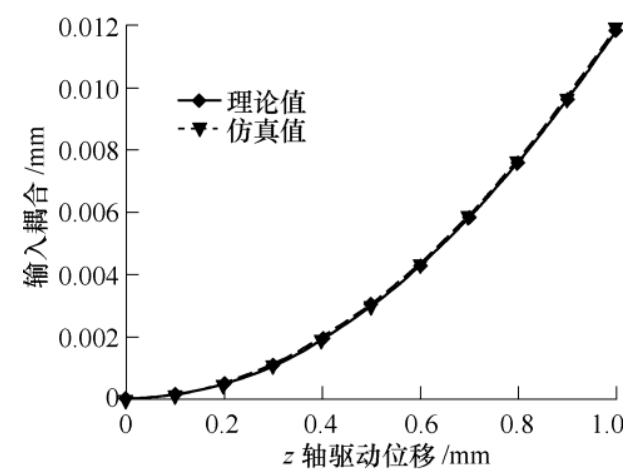

(b) $x$ 轴输入点的耦合位移

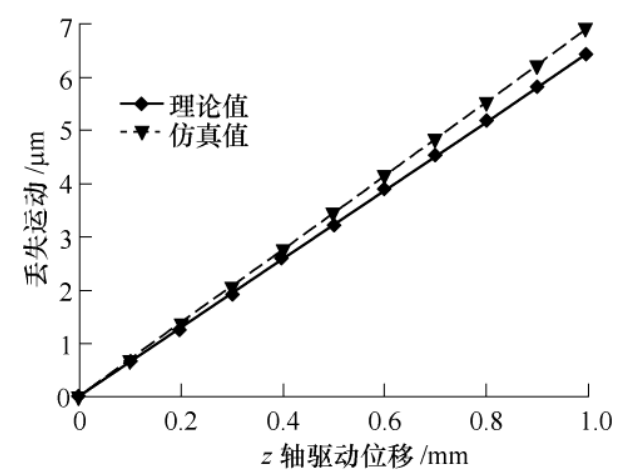

(c) $z$ 轴方向的丢失运动

图 $17 z$ 轴方向平台静态性能验证

图 17a 所示为反作用力 $F_{z}$ 的理论(式(37))、仿真 值曲线, 最大值分别为 $80.23 \mathrm{~N} 、 79.06 \mathrm{~N}$, 在 $0 \sim$ $1 \mathrm{~mm}$ 驱动位移内, 理论与仿真之间的误差为 $0.5 \% \sim 3.7 \%$ 。
图 $17 \mathrm{~b}$ 所示为 $x$ 轴输入点处耦合位移 $\delta_{x}^{+}$的理 论(式(35))、仿真值曲线, 最大值分别为 $11.81 \mu \mathrm{m}$ 、 $11.92 \mu \mathrm{m}$, 小于驱动位移的 $1.2 \%$ 。在 $0 \sim 1 \mathrm{~mm}$ 驱动位移内, 理论与仿真之间的误差为 $0.9 \%$ $11.7 \%$ 。

图 $17 \mathrm{c}$ 所示为丢失运动 $\delta_{z l o}$ 的理论 (式 (39))、仿真值曲线, 最大值分别为 $6.46 \mu \mathrm{m}$ 、 $6.92 \mu \mathrm{m}$, 小于驱动位移的 $0.7 \%$ 。在 $0 \sim 1 \mathrm{~mm}$ 驱动位移内, 理论与仿真之间的误差为 $6.8 \% \sim 7.2 \%$, 丢失运动仿真值并未发生明显 突变, 表明细长杆无屈曲现象。

根据仿真结果, 结构中最大应力为 $121 \mathrm{MPa}$ 小 于材料许用应力, 动平台中心点在 $x, y$ 轴方向无耦 合位移, 输出完全解耦。

\section{3 动态特性验证}

平台的 1 6 阶模态振型如图 18 所示, 固有 频率的理论(式(52))、仿真值及二者相对误差如表 3 所示。

由图 18、表 3 可知平台 $1 \sim 3$ 阶(移动自由度方 向)固有频率, 与 $4 \sim 6$ 阶固有频率差别明显, 表明 平台动态性能良好, 在非自由度方向具有较强的抗 干扰性能。

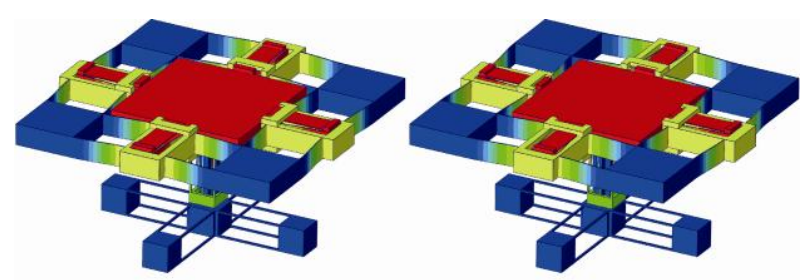

(a) 一阶振型

(b) 二阶振型

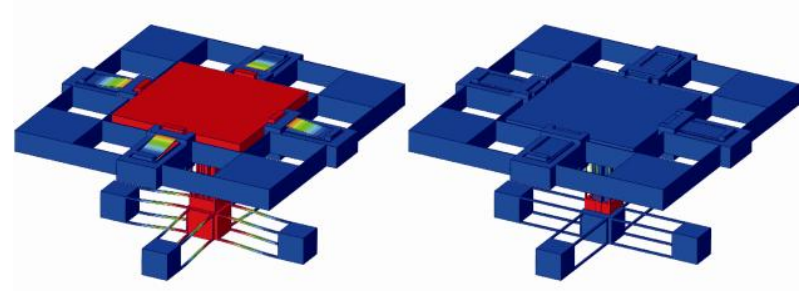

(c) 三阶振型

(d) 四阶振型

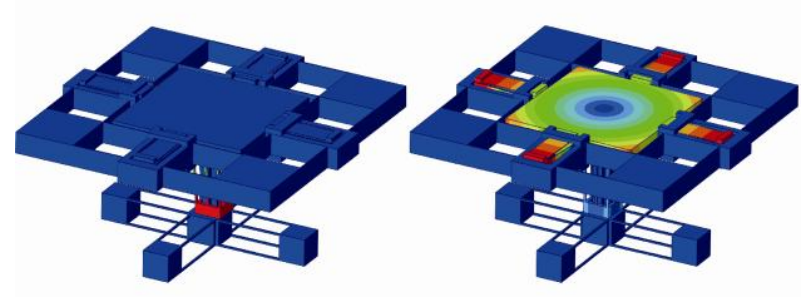

(e) 五阶振型

(f) 六阶振型

图 18 平台的 $1 \sim 6$ 阶模态振型 
表 3 平台固有频率的理论、仿真值及相对误差

\begin{tabular}{cccc}
\hline 阶数 & 理论值 $/ \mathrm{Hz}$ & 仿真值 $/ \mathrm{Hz}$ & 相对误差 $(\%)$ \\
\hline 1 & 50.22 & 49.56 & 1.3 \\
2 & 50.22 & 49.56 & 1.3 \\
3 & 59.73 & 59.39 & 0.6 \\
4 & - & 208.78 & - \\
5 & - & 208.83 & - \\
6 & - & 237.67 & - \\
\hline
\end{tabular}

通过上述对平台静、动态特性的逐一验证, 表 明了理论模型的正确性与参数化设计平台性能的优 越性。

\section{5 结论}

(1) 提出了两种新型的多自由度大行程柔性运 动副, 并给出了三种新型的大行程柔性微定位平台 设计实例。

(2) 采用非线性模型法和柔度矩阵法建立了平 台的静、动态特性理论模型, 基于该模型确定了平 台的最优参数, 实现了平台的参数化设计。

(3) 通过有限元仿真对理论模型进行了验证, 根据 理论与仿真结果, 平台一阶固有频率为 $49.6 \mathrm{~Hz}$, 在 $1 \mathrm{~mm}$ 运动行程内, 输入耦合小于 $1.2 \%, x 、 z$ 轴丢失 运动分别小于 $0.32 \% 、 0.7 \%$ 且输出完全解耦。

(4) 后续将搭建试验平台, 对理论模型及平台 的相关性能做进一步的测试与验证。

\section{参 考 文 献}

[1] HOWELL L L. Compliant mechanisms[M]. New York:

John Wiley and Sons, 2001.

[2] 于靖军, 郝广波, 陈贵敏, 等. 柔性机构及其应用研 究进展 $[\mathrm{J}]$. 机械工程学报，2015，51(13)：53-68.

YU Jingjun, HAO Guangbo, CHEN Guimin, et al. Stateof-art of compliant mechanisms and their applications[J]. Journal of Mechanical Engineering, 2015, 51(13): 53-68.

[3] YONG Y K , MOHEIMANI S O R , KENTON B J , et al. Invited review article: High-speed flexure-guided nanopositioning: Mechanical design and control issues[J]. Review of Scientific Instruments, 2012, 83(12): 121101.

[4] ZHU Z, TO S, ZHU W L, et al. Optimum design of a piezo-actuated triaxial compliant mechanism for nanocutting[J]. IEEE Transactions on Industrial Electronics, 2018, 65(8): 6362-6371.

[5] LIN C, WU Z H, REN Y H, et al. Characteristic analysis of unidirectional multi-driven and large stroke micro- /nano-transmission platform[J]. Microsystem Technologies, 2017, 23(8): 3389-3400.

[6] WATANABE S, ANDO T. High-speed $X Y Z$ nanopositioner for scanning ion conductance microscopy[J]. Applied Physics Letters, 2017, 111(11): 113106.

[7] 李海洋, 郝广波, 于靖军, 等. 空间平动柔性并联机 构的系统设计方法研究[J]. 机械工程学报, 2018, 54(13): 57-65.

LI Haiyang, HAO Guangbo, YU Jingjun. et al. Systematic approach to the design of spatial translational compliant parallel mechanisms[J]. Journal of Mechanical Engineering, 2018, 54(13): 57-65.

[8] HAO G B, LI H Y. Design of 3-legged $X Y Z$ compliant parallel manipulators with minimized parasitic rotateons[J]. Robotica, 2015, 33(4): 787-806.

[9] TANG X Y, CHEN I M, LI Q. Design and nonlinear modeling of a large-displacement $X Y Z$ flexure parallel mechanism with decoupled kinematic structure[J]. Review of Scientific Instruments， 2006，77(11): 115101-115111.

[10] LI Y M, XU Q S. Design and optimization of an XYZ parallel micromanipulator with flexure hinges[J]. Journal of Intelligent and Robotic Systems, 2009, 55(4-5): 377-402.

[11] LI Y M, XU Q S. A totally decoupled piezo-driven $X Y Z$ flexure parallel micropositioning stage for micro/nanomanipulation[J]. IEEE Transactions on Automation Science and Engineering, 2011，8(2): 265-279.

[12] YUE Y, GAO F, ZHAO X, et al. Relationship among input-force payload stiffness and displacement of a 3-DOF perpendicular parallel micro-manipulator[J]. Mechanism and Machine Theory, 2010, 45(5): 756-771.

[13] LI H Y, HAO G B, RICHARD C, et al. A new $X Y Z$ compliant parallel mechanism for nano-manipulation: Design and analysis[J]. Micromachines, 2016, 7(2): 23.

[14] ZHANG X Z, XU, Q S. Design, fabrication and testing of a novel symmetrical 3-DOF large-stroke parallel micro/nano-positioning stage[J]. Robotics and ComputerIntegrated Manufacturing, 2018，54: 162-172.

[15] HAO G B, KONG X W. Design and modeling of a large-range modular $X Y Z$ compliant parallel manipulator using identical spatial modules[J]. Journal of Mechanisms and Robotics, 2012，4(2): 021009.

[16] LI Y M, WU Z G. Design, analysis and simulation of a novel 3-DOF translational micromanipulator based on the PRB model[J]. Mechanism and Machine Theory, 2016, 
100: $235-258$.

[17] KOSEKI Y, TANIKAWA T, KOYACHI $\mathrm{N}$, et al. Kinematic analysis of translational 3-DOF micro parallel mechanism using matrix method[J]. IEEE/RSJ International Conference on Intelligent Robots and Systems, 2000, 1(3): 786-792.

[18] TANG H, LI Y M. Design analysis and test of a novel 2-DOF nanopositioning system driven by dual mode[J]. IEEE Transactions on Robotics, 2013， 29(3): 650-662.

[19] AWTAR S, PARMAR G. Design of a large range $X Y$ nanopositioning system[J]. Journal of Mechanisms and Robotics, 2013, 5(2): 021008.

[20] MA F L, CHEN G M. Bi-BCM: A closed-form solution for fixed-guided beams in compliant mechanisms[J]. Journal of Mechanisms and Robotics, 2017, 9(1): 014501.
[21] HERPE X, WALKER R, DUNNIGAN M, et al. On a simplified nonlinear analytical model for the characterisation and design optimisation of a compliant $X Y$ micromotion stage[J]. Robotics and Computer- Integrated Manufacturing, 2018, 49: 66-76.

[22] 杨志军, 白有盾, 陈新, 等. 基于应力刚化效应的动 态特性可调微动平台设计新方法 $[\mathrm{J}]$. 机械工程学报, 2015, 51(23): 153-159.

YANG Zhijun, BAI Youdun, CHEN Xin, et al. A new design method of dynamic characteristics adjustable micro motion stage based on tension stiffening[J]. Journal of Mechanical Engineering, 2015， 51(23): 153-159.

作者简介: 曹毅(通信作者), 男, 1974 年出生, 博士, 教授, 硕士研究 生导师。主要研究方向为机器人机构学。

E-mail: caoyi@jiangnan.edu.cn 